\title{
AEROSOL PRODUCTS FROM THE FUTURE SPACE LIDAR AEOLUS
}

\author{
Pauline Martinet $^{1}$ *, Alain Dabas ${ }^{1}$, Vincent Lever ${ }^{1}$, Pierre Flamant $^{2}$, Dorit Huber ${ }^{3}$ \\ ${ }^{1}$ Météo-France, CNRM-GAME/GMEI,Toulouse, France*Email: pauline.martinet@meteo.fr \\ ${ }^{2}$ CNRS, Laboratoire de Météorologie Dynamique, Palaiseau, France \\ ${ }^{3}$ DoRit, Munich, Germany
}

\begin{abstract}
Ready for launch by the end of 2016, the Doppler lidar mission AEOLUS from the European Space Agency (ESA) will be the first High-Spectral Resolution Lidar (HSRL) in space. Operating in the UV, it implements two detection channels for aerosol and molecular backscatter. The system is primarily designed for the measurement of winds, but the HSRL capability enables the measurement of the particulate backscatter and extinction coefficients without any a priori assumption on the aerosol type. The level-2A (L2A) processor has been developed for these measurements and tested with synthetic data. The results show good aerosol backscatter profiles can be retrieved. Extinction coefficients are reasonable but do not reach the quality of backscatter coefficients. A precise, full, radiometric calibration of the lidar is required. A major limitation of the system is a single polarization component of the light is detected leading to an underestimation of backscatter coefficients when the atmospheric particles are depolarizing. The vertical resolution goes from 250 meters in the lowest part of the atmosphere, to $2 \mathrm{~km}$ in the lower stratosphere. The maximum altitude can reach above $20 \mathrm{~km}$. The basic horizontal averaging is $90 \mathrm{~km}$. Averaging on shorter distances (down to a few $\mathrm{km}$ ) are possible but require a sufficient signal to noise ratio.
\end{abstract}

\section{INTRODUCTION}

The space-based Doppler mission AEOLUS of the European Space Agency should be ready for launch in the late 2016 or early 2017. It should be the first Doppler lidar in space able to measure winds over the globe throughout the entire depth of the atmosphere (from the ground up to $25 \mathrm{~km}$ or $30 \mathrm{~km}$ ). Wind measurements will be delivered in quasi real-time and assimilated by numerical weather prediction systems of meteorological services. They should improve the quality of weather forecasts, in particular in the tropics and at high altitudes where wind observations are otherwise scarce. The single instrument aboard AEOLUS is the directdetection Doppler lidar ALADIN. ALADIN operates a UV laser emitting $80 \mathrm{~mJ}$ pulses at the rate of $50 \mathrm{~s}^{-1}$. It implements two sequential detection channels. The light is first passed through a narrow-band Fizeau interferometer, and the reflection on the Fizeau is transmitted through a large-band, double Fabry-Pérot. The Fizeau resolves the narrow-band spectrum of the aerosol backscatter, while the contrast of the light fluxes transmitted through the two FPs is converted into a wind Doppler shift as proposed in the late 80 s by Chanin et al. [3]. ALADIN is thus a High-Spectral-Resolution Lidar (HSRL). Although it has been designed for wind measurements, the signals it detects bear information on the optical properties of atmospheric particles, either aerosols or hydrometeors. As demonstrated in [4] and [5], this information can be retrieved without making any a priori assumption on the aerosol Backscatter-to-Extinction Ratio (BER). Here the BER is measured by the lidar that can then not only quantify the aerosol properties of the atmosphere but also give a qualitative information on the aerosol type. This kind of information is very interesting for the scientific studies on the impact of aerosols on climate 
change and could also be assimilated by numerical models predicting air quality on a global scale as it is done in the frame of the European GMES-MACC service (see http://www.gmes-atmosphere.eu).

\section{METHODOLOGY}

The spectrum of the light received by the lidar consists of a broad spectrum of molecular backscatter and a narrow spectrum of particle backscatter. It passes through 3 interferometers. One is a narrow band Fizeau filter, the two others are broad-band Fabry-Perot filters located on either side of the laser frequency. The power transmitted through the Fizeau $\left(\mathrm{S}_{\mathrm{Fiz}}\right)$ and the sum of the powers transmitted through the two Fabry-Pérot filters $\left(\mathrm{S}_{\mathrm{FP}}\right)$ can be written:

$$
\begin{aligned}
& S_{F P}(z)= \\
& \frac{E_{0}}{R(z)^{2}}\left[C_{1} \beta_{m o l}(z)+\right. \\
& \left.C_{2} \beta_{\text {aer }}(z)\right] \exp \left(-2 \int_{z}^{+\infty}\left(\alpha_{\text {aer }}(x)+\right.\right. \\
& \left.\left.\alpha_{m o l}(x)\right) d R(x)\right) \\
& S_{F i z}(z)= \\
& \frac{E_{0}}{R(z)^{2}}\left[C_{3} \beta_{\text {aer }}(z)+\right. \\
& \left.C_{4} \beta_{\text {mol }}(z)\right] \exp \left(-2 \int_{z}^{+\infty}\left(\alpha_{a e r}(x)+\right.\right. \\
& \left.\left.\alpha_{\text {mol }}(x)\right) d R(x)\right)
\end{aligned}
$$

Here, $E_{0}$ is the emitted laser energy, $R(z)$ is the distance from the satellite to the altitude $z$ along the line-of-sight, $\beta_{\text {mol }}$ and $\beta_{a e r}$ are the molecular and aerosol backscatter coefficients, $\alpha_{m o l}$ and $\alpha_{a e r}$ are the molecular and aerosol extinction coefficients, and $C_{1}, C_{2}, C_{3}$ and $C_{4}$ are calibration constants. The signals recorded by the lidar are integrated in 24 height-bins $\left[z_{k}, z_{k}+1\right]$ of thicknesses varying from $250 \mathrm{~m}$ in the lowest part of the atmosphere to $2 \mathrm{~km}$ in the lower stratosphere:

$$
\begin{gathered}
S_{F P}(k)=\int_{z_{k}}^{z_{k+1}} S_{F P}(z) d z \\
S_{F i z}(k)=\int_{z_{k}}^{z_{k+1}} S_{F i z}(z) d z
\end{gathered}
$$

Due to the transmission characteristics of the detectors, $C_{2}<C_{1}$ and $C_{4}<C_{3}$, the molecular and aerosol backscatter contributions can be separated after a crosstalk correction:

$$
\begin{array}{r}
X(k) \approx \frac{1}{R\left(z_{k}\right)^{2}} \int_{z_{k}}^{z_{k+1}} \beta_{m o l}(z) \exp \left(-2 \int_{z}^{+\infty}\left(\alpha_{a e r}(x)+\right.\right. \\
\left.\left.\alpha_{m o l}(x)\right) d R(x)\right) d z=\frac{1}{E_{0}} \frac{C_{2} S_{F P}(k)-C_{3} S_{F i z}(k)}{C_{2} C_{4}-C_{1} C_{4}} \\
Y(k) \approx \frac{1}{R\left(z_{k}\right)^{2}} \int_{z_{k}}^{z_{k+1}} \beta_{a e r}(z) \exp \left(-2 \int_{z}^{+\infty}\left(\alpha_{a e r}(x)+\right.\right. \\
\left.\left.\alpha_{m o l}(x)\right) d R(x)\right) d z=\frac{1}{E_{0}} \frac{C_{4} S_{F i z}(k)-C_{1} S_{F P}(k)}{C_{2} C_{4}-C_{1} C_{4}}
\end{array}
$$

The operation requires a good calibration of the lidar. The molecular backscatter $\beta_{m o l}$ and extinction $\alpha_{m o l}$ are known functions of the air density that a numerical weather prediction model can predict with a good precision. It therefore remains two unknowns: the height-bin average aerosol backscatter $\hat{\beta}_{a e r}$ and the heightbin average aerosol extinction $\hat{\alpha}_{a e r}(k)$. Both can thus be determined for each height-bin.

As the receiver is transmitting a single polarization parallel to the laser emission, the backscatter coefficients above are for this single polarization.

\section{RESULTS}

When the molecular and aerosol backscatter contributions have been separated, the heightbin average aerosol properties can be retrieved. The L2A processor provides users with several products derived from the backscatter and the extinction coefficients: local optical depth, scattering ratio, backscatter-to-extinction ratio. In order to evaluate the performance of the chain of ADM-Aeolus processors, an end-to-end simulator (E2S) has been developed by ESA to simulate the Rayleigh and Mie signals received in the double Fabry-Pérot and the Fizeau interferometers. The L2A processor has been evaluated for different atmospheric scenarios from simple to complex scenes observed during the NASA's LITE (Lidar In-space Technology Experiment) experiment. By comparing the aerosol properties retrieved by the L2A to the 
input profiles used in the E2S to simulate the lidar signals, the expected accuracy of the aerosol properties can be studied. The L2A processor is expected to retrieve the backscatter coefficient and the scattering ratio with a good accuracy in cirrus clouds and boundary layers. Relative deviations from the true values are within 20 to $50 \%$ with an average of $20 \%$ in cirrus clouds (figure 2) and 20 to $45 \%$ in the boundary layer. Oscillations between overestimation and underestimation are observed in the retrieval of the extinction coefficient due to the iterative algorithm used to derive this parameter. However, the overall shape of the aerosol and cloud layers is well captured by the L2A. Relative deviations for the extinction are within 20 to $60 \%$ in cirrus layers (figure 1) and boundary layer aerosols with an average error of $45 \%$ in layers with an extinction coefficient higher than approximately $1.10^{-4} \mathrm{~m}^{-1}$. For small values of extinction, retrieval errors up to $80 \%$ or more are observed. The retrieval inside and below opaque clouds is limited by the strong attenuation of the lidar signal (figures 1 and 2).

\section{CONCLUSIONS}

The on-going developments on the L2A processor have shown the potential of ADMAeolus to retrieve aerosol and cloud properties. With a launch scheduled in 2017, ADM-Aeolus could fill in the gap between the CALIPSO and EarthCare missions. ADM-Aeolus will also be the first satellite platform to provide information on both cloud microphysical properties and cloud dynamics. An accuracy of approximately $20 \%$ in backscatter and $45 \%$ in extinction can be expected in clouds and boundary layer aerosols. For the moment, these results are obtained with a horizontal averaging of $90 \mathrm{~km}$. In the future, a feature finder algorithm, aiming at improving the horizontal resolution of the L2A by retrieving the aerosol properties at a smaller horizontal scale will be tested. This averaging at shorter distance requires a good signal-to-noise ratio.

\section{ACKNOWLEDGEMENT}

The work described in the paper was funded by the European Space Agency in the frame of the AEOLUS mission. We would like to thank ESA team - namely Frank de Bruin, Anne Straume, and Thomas Kanitz - and the other members of the level 1/level 2A development teams for their advice, support and feedbacks.

\section{REFERENCES}

[1] Regan, M. J., X. Wang, M. J. Osborn, 2002: Spaceborne lidar calibration from cirrus and molecular backscatter returns, IEEE Trans. Geosci. Remote Sens., 40, 2285-2290.

[2] O'Connor, E. J., A. J. Illingworth, R. J. Hogan, 2004: A technique for autocalibration of cloud lidar, J. Atmos. Ocean Tech., 21(5), 777-778.

[3] Chanin, M. L., A. Garnier, A. Hauchecorne, J. Porteneuve, 1989: A Doppler lidar for measuring winds in the middle atmosphere. Geophysical Research Letters, 16, 12731276.

[4] Ansmann, A., U. Wandinger, O. Le Rille, D. Lajas, A. G. Straume, 2007: Particle backscatter and extinction profiling with the spaceborne high-spectral-resolution Doppler lidar ALADIN: methodology and simulations. Applied Optics, 46, 6606-6622.

[5] Flamant, P., Cuesta, J., Denneulin, M.-L., Dabas, A. and Huber, D., 2008: ADMAeolus retrieval algorithms for aerosol and cloud products. Tellus, 60A, 273-288.

[6] Horanyi, A. et al. (2014) The assimilation of horizontal line-of-sight wind information into the ECMWF data assimilation and forecasting system, Part I: The assessment of wind impact, Q. J. R. Meteorol. Soc. (2014) 

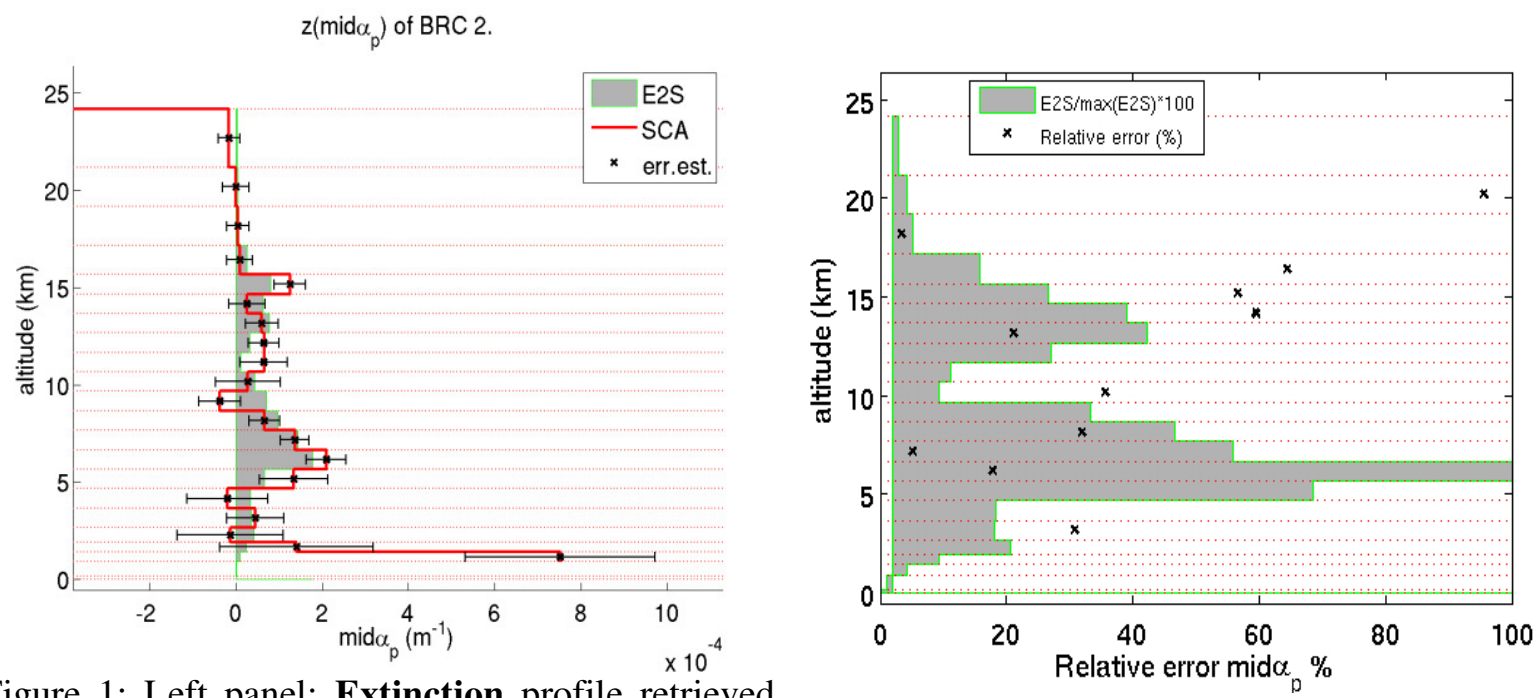

Figure 1: Left panel: Extinction profile retrieved by the L2A (red line) compared to the E2S input profile (gray area). The estimation of the retrieval uncertainties mainly due to the photon counting noise is shown for each layer as horizontal black line. Right panel: Relative deviation between the L2A retrieval and the E2S input profile (black crosses). The ratio of the E2S extinction value to the maximum value found over the profile is also displayed in gray area.
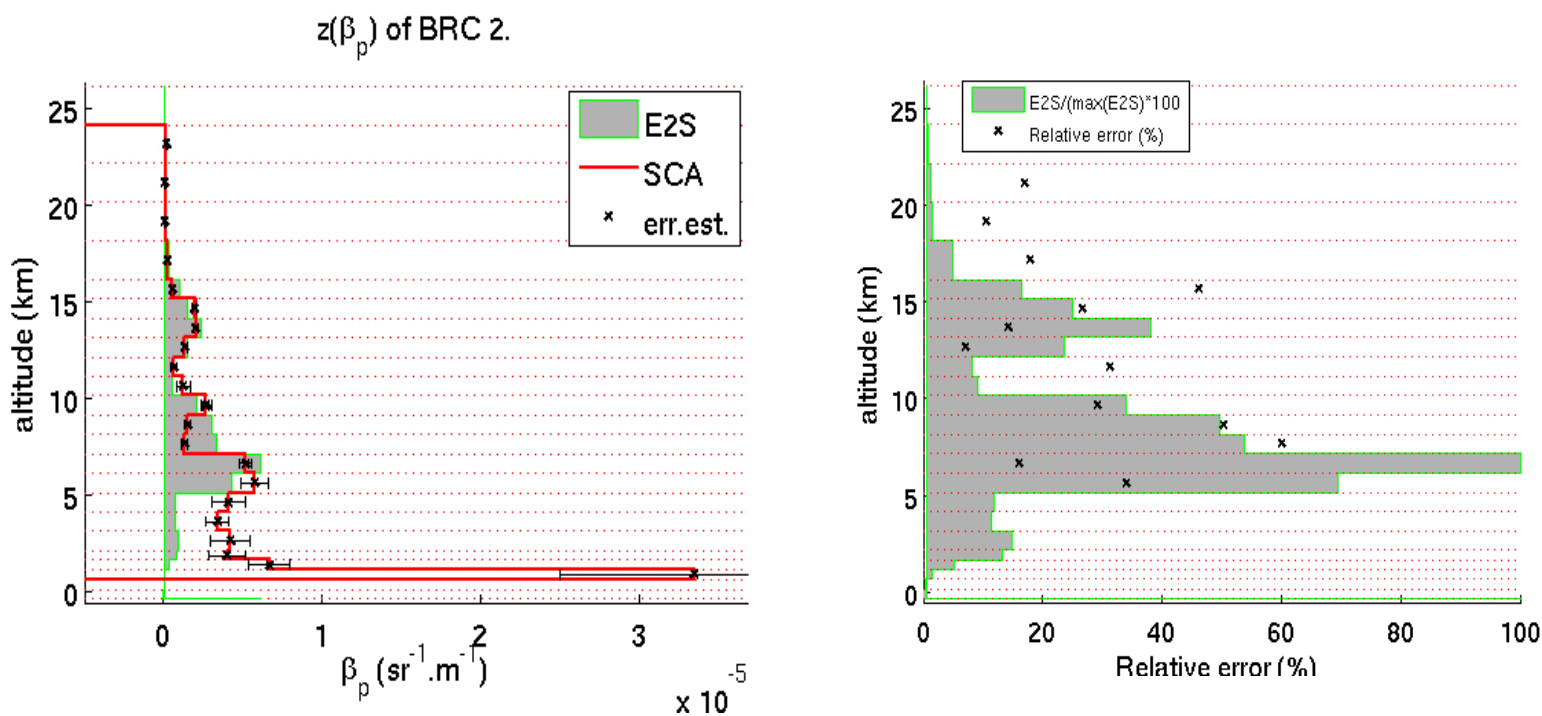

Figure 2: Left panel: Backscatter profile retrieved by the L2A (red line) compared to the E2S input profile (gray area). The estimation of the retrieval uncertainties mainly due to the photon counting noise is shown for each layer as horizontal black line. Right panel: Relative deviation between the L2A retrieval and the E2S input profile (black crosses). The ratio of the E2S backscatter value to the maximum value found over the profile is also displayed in gray area. 